\title{
Approximation properties of mixed sampling-Kantorovich operators
}

\author{
Laura Angeloni $^{1}$ (D) $\cdot$ Danilo Costarelli ${ }^{1} \cdot$ Gianluca Vinti $^{1}$
}

Received: 24 February 2020 / Accepted: 17 September 2020 / Published online: 11 October 2020

(c) The Author(s) 2020

\begin{abstract}
In the present paper we study the pointwise and uniform convergence properties of a family of multidimensional sampling Kantorovich type operators. Moreover, besides convergence, quantitative estimates and a Voronovskaja type theorem have been established.
\end{abstract}

Keywords Sampling-Kantorovich operators · Pointwise convergence $\cdot$ Uniform convergence $\cdot$ Order of approximation - Asymptotic expansions · Voronovskaja formulae

Mathematics Subject Classification 41A30 - 41A05

\section{Introduction}

In the last years there was an increasing interest in approximation by means of families of discrete operators in several function spaces both in the one-dimensional case and in multidimensional setting, also thanks to the applicative outcome of such results (see, e.g., $[18,27,28,36,37,41])$. For example, the generalized sampling series defined as

$$
\left(S_{w} f\right)(\mathrm{t})=\sum_{\mathrm{k} \in \mathbb{Z}^{N}} f\left(\frac{\mathrm{k}}{w}\right) \chi(w \mathrm{t}-\mathrm{k}),
$$

$t \in \mathbb{R}^{N}, w>0$, where $\chi$ is a kernel, have been widely studied with respect to several notions of convergence, such as pointwise, uniform, $L^{p}$, modular convergence [23-25] and also, recently [5,7], convergence in variation (for other approximation results in BV-spaces see, e.g., [9-13]). The interest of such operators is also due to their deep connections with problems of Signal and Image Processing: indeed they furnish an approximate version of

Laura Angeloni

laura.angeloni@unipg.it

Danilo Costarelli

danilo.costarelli@unipg.it

Gianluca Vinti

gianluca.vinti@unipg.it

1 Department of Mathematics and Computer Science, University of Perugia, Via Vanvitelli 1, 06123

Perugia, Italy 
the Shannon Sampling Theorem (see, e.g., $[46,47])$ that allows to reconstruct not necessarily band-limited signals (images).

In order to obtain convergence in variation for the operators ( $\star$ ), in [7] a new family of discrete operators has been introduced:

$$
\left(K_{w, j} f\right)(\mathrm{t}):=\sum_{\mathrm{k} \in \mathbb{Z}^{N}}\left[w \int_{\frac{k_{j}}{w}}^{\frac{k_{j}+1}{w}} f\left(\frac{k_{1}}{w}, \ldots, u \ldots, \frac{k_{N}}{w}\right) d u\right] \chi(w \mathrm{t}-\mathrm{k}),
$$

for every $t \in \mathbb{R}^{N}, w>0$ and $j=1, \ldots, N$. The operators $(*)$, that here will be called mixed sampling-Kantorovich operators, are essentially a Kantorovich version of the generalized sampling series where the integral mean is computed on just one section of the function involved, while the usual Kantorovich version replaces the whole value $f\left(\frac{\mathrm{k}}{w}\right)$ with an integral mean on a multidimensional interval around the sampling node $\frac{\mathrm{k}}{w}$, i.e.,

$$
\left(K_{w} f\right)(\mathrm{t}):=\sum_{\mathrm{k} \in \mathbb{Z}^{N}}\left[w^{N} \int_{\prod_{j=1}^{N}\left[\frac{k_{j}}{w}, \frac{k_{j}+1}{w}\right]} f(\mathrm{u}) d \mathrm{u}\right] \chi(w \mathrm{t}-\mathrm{k}),
$$

for every $t \in \mathbb{R}^{N}, w>0$.

The introduction of the operators $(*)$ was naturally motivated by the fact that such operators allow to obtain a multidimensional generalization of the classical relation proved by Lorentz among the derivative of the Bernstein polynomials and the Kantorovich polynomials acting on the derivative of the function, in the one-dimensional case: in particular, in [7] it is proved that the $j$-th partial derivative of the generalized sampling series $\frac{\partial S_{w} f}{\partial x_{j}}$ coincides with a combination of shifted mixed sampling-Kantorovich operators $\left(K_{w, j} \frac{\partial f}{\partial x_{j}}\right)$ acting on the $j$-th partial derivative of $f$ in case of kernels of averaged type.

Nevertheless, the family of operators $(*)$ appear as an interesting intermediate case between the generalized sampling series $(\star)$ and the classical Kantorovich operators $(* *)$ and therefore the approximation results that we will present cannot be derived by the analogous results that have been previously obtained in the literature for the operators $(\star)$ or $(* *)$.

We recall that the study of the approximation properties of the Kantorovich version of families of operators is a widely investigated topic in the literature. Just to mention some examples, in [3,35] approximation results of the Stancu-Kantorovich operators based on Polya-Eggenberger distribution are presented; in [40] it is established an inverse result for bivariate Kantorovich type sampling series and for their generalized Boolean sum (see also, e.g., $[42,43,45]$ for some modifications and generalizations), while in [4] the rate of convergence of perturbed Kantorovich-Choquet univariate and multivariate normalized neural network operators with respect to the uniform norm is obtained. About the Kantorovich version of the generalized sampling series $(* *)$, results about pointwise and uniform convergence, $L^{p}$-convergence, modular convergence, rate of approximation and inverse results have been obtained (see, e.g, [6,15,26,28-33].

It is well-known that approximation by means of the Kantorovich version of the sampling operators presents several advantages with respect to the class $\left\{S_{w} f\right\}_{w>0}$, also from the point of view of the applications to Signal Processing (see, e.g., $[15,39,44])$, due to the presence of the integral mean. For instance, this allows to reduce the so-called time-jitter error that occurs when, in the practice, the sampled values are not computed exactly over the sample nodes. Nevertheless, since the mixed operators $(*)$ appear to be an intermediate class among the sampling series and the Kantorovich operators, they can be applied in situations in which, in 
the approximation process, it is more suitable to keep the value of the function on the sample nodes except just one direction, where the integral mean is computed. For example, this can be useful in situations in which the interest is to reduce time-jitter error in just one direction to guarantee a good reconstruction saving computational operations with respect to the usual sampling-Kantorovich operators.

In [7] convergence in $L^{p}\left(\mathbb{R}^{N}\right)$ for the family of operators $(*)$ is proved. Here we give results about pointwise and uniform convergence. Moreover, we establish a quantitative estimate in terms of the modulus of continuity of the function that, as a consequence, gives a result about the order of approximation if the function belongs to a Lipschitz class. Finally, we prove an asymptotic expansion for $\left(K_{w, j} f\right)$ that allows us to obtain a Voronovskaja type theorem.

\section{Notations and preliminaries}

We will denote by $C\left(\mathbb{R}^{N}\right)$ the space of all the bounded and uniformly continuous functions $f: \mathbb{R}^{N} \rightarrow \mathbb{R}$ endowed with the supremum norm $\|f\|_{\infty}$.

We will study the family of mixed sampling-Kantorovich operators recently introduced in [7], defined as

$$
\left(K_{w, j} f\right)(\mathrm{t}):=\sum_{\mathrm{k} \in \mathbb{Z}^{N}}\left[w \int_{\frac{k_{j}}{w}}^{\frac{k_{j}+1}{w}} f\left(\frac{k_{1}}{w}, \ldots, u \ldots, \frac{k_{N}}{w}\right) d u\right] \chi(w \mathrm{t}-\mathrm{k}),
$$

for every $t \in \mathbb{R}^{N}, w>0$ and $j=1, \ldots, N$.

Here $\chi$ is a kernel, that is, a function $\chi: \mathbb{R}^{N} \rightarrow \mathbb{R}$ that satisfies the following assumptions:

$\left(\chi_{1}\right) \quad \chi$ is continuous and such that $\sum_{\mathrm{k} \in \mathbb{Z}^{N}} \chi(\mathrm{t}-\mathrm{k})=1$, for every $\mathrm{t} \in \mathbb{R}^{N}$;

$\left(\chi_{2}\right) M_{0}(\chi):=\sup _{\mathrm{u} \in \mathbb{R}^{N}} \sum_{\mathrm{k} \in \mathbb{Z}^{N}}|\chi(\mathrm{u}-\mathrm{k})|<+\infty$, where the convergence of the series $\sum_{\mathrm{k} \in \mathbb{Z}^{N}}|\chi(\mathrm{u}-\mathrm{k})|$ is uniform on the compact subsets of $\mathbb{R}^{N}$.

We point out that above assumptions are standard working with discrete families of operators (see, e.g., $[7,8,17,20,21])$ and it is easy to provide examples of kernels that fulfill them. Among them, for example we can mention the multivariate version of the Jackson, Fejér, and central B-spline (product) kernels, or the well-known Bochner-Riesz (radial) kernels. For more details, see, e.g., [16,19,22,30,34,38,48].

It is easy to see that mixed sampling-Kantorovich operators $(*)$ are well-defined if, for example, $f$ is bounded. Indeed, if $|f(t)| \leq L$, for every $t \in \mathbb{R}^{N}$,

$$
\begin{aligned}
\left|\left(K_{w, j} f\right)(\mathrm{t})\right| & \leq \sum_{\mathrm{k} \in \mathbb{Z}^{N}}\left[w \int_{\frac{k_{j}}{w}}^{\frac{k_{j}+1}{w}}\left|f\left(\frac{k_{1}}{w}, \ldots, u \ldots, \frac{k_{N}}{w}\right) d u\right|\right]|\chi(w \mathrm{t}-\mathrm{k})| \\
& \leq L \sum_{\mathrm{k} \in \mathbb{Z}^{N}}|\chi(w \mathrm{t}-\mathrm{k})| \leq L M_{0}(\chi)<+\infty,
\end{aligned}
$$

by $\left(\chi_{2}\right)$, for every $t \in \mathbb{R}^{N}, w>0, j=1, \ldots, N$.

We finally recall the following multidimensional notations that we will use in the paper: for a function $f: \mathbb{R}^{N} \rightarrow \mathbb{R}$ and $\mathrm{x}=\left(x_{1}, \ldots, x_{N}\right) \in \mathbb{R}^{N}$, we will write

$$
\mathrm{x}_{j}^{\prime}=\left(x_{1}, \ldots, x_{j-1}, x_{j+1}, \ldots, x_{N}\right) \in \mathbb{R}^{N-1}, \quad \mathrm{x}=\left(\mathrm{x}_{j}^{\prime}, x_{j}\right), \quad f(\mathrm{x})=f\left(\mathrm{x}_{j}^{\prime}, x_{j}\right),
$$

to emphasize the $j$-th coordinate of $\mathrm{x}, j=1, \ldots, N$ and, for $\mathrm{x} \in \mathbb{R}^{N}$ and $\alpha \in \mathbb{R}$, we will use the usual notation for products and quotients, i.e., $\alpha \mathrm{x}=\left(\alpha x_{1}, \ldots, \alpha x_{N}\right)$ and, for $\alpha \neq 0$, 
$\frac{\mathrm{x}}{\alpha}=\left(\frac{x_{1}}{\alpha}, \ldots, \frac{x_{N}}{\alpha}\right)$. Finally, $[\mathrm{x}]=\left(\left[x_{1}\right], \ldots,\left[x_{N}\right]\right)$ will denote the integer part of $\mathrm{x} \in \mathbb{R}^{N}$, $\|\mathrm{x}\|$ will denote the usual Euclidean norm of $\mathbb{R}^{N}$, and $\mathrm{x}^{\mathrm{Y}}=\prod_{i=1}^{N} x_{i}^{y_{i}}, \mathrm{x}, \mathrm{y} \in \mathbb{R}^{N}$, when the power is well-defined.

\section{Pointwise and uniform convergence}

We will first prove pointwise convergence for the mixed sampling-Kantorovich operators $\left\{K_{w, j} f\right\}_{w>0}, j=1, \ldots, N$.

Theorem 1 If $f: \mathbb{R}^{N} \rightarrow \mathbb{R}$ is bounded, then, for every $j=1, \ldots, N$,

$$
\lim _{w \rightarrow+\infty}\left(K_{w, j} f\right)\left(t_{0}\right)=f\left(t_{0}\right),
$$

at each point $t_{0} \in \mathbb{R}^{N}$ where $f$ is continuous.

Proof Let $t_{0} \in \mathbb{R}^{N}$ be a point of continuity for $f$ and let us fix $j=1, \ldots, N$ and $\epsilon>0$.

Let $\delta>0$ be such that, for every $t \in \mathbb{R}^{N}$ for which $\left\|t-t_{0}\right\|<\delta$,

$$
\left|f(t)-f\left(t_{0}\right)\right|<\frac{\epsilon}{2 M_{0}(\chi)},
$$

where $M_{0}(\chi)$ is the absolute moment of order 0 of $\chi$ of assumption $\left(\chi_{2}\right)$. By $\left(\chi_{1}\right)$ there holds, for $w>0$,

$$
\begin{aligned}
& \left|\left(K_{w, j} f\right)\left(\mathrm{t}_{0}\right)-f\left(\mathrm{t}_{0}\right)\right| \leq \\
& \quad \leq\left\{\sum_{\left\|\mathrm{k}-w \mathrm{t}_{0}\right\| \leq \frac{w \delta}{2}}+\sum_{\left\|\mathrm{k}-w \mathrm{t}_{0}\right\|>\frac{w \delta}{2}}\right\}\left\{w \int_{\frac{k_{j}}{w}}^{\frac{k_{j}+1}{w}}\left|f\left(\frac{k_{1}}{w}, \ldots, u \ldots, \frac{k_{N}}{w}\right)-f\left(\mathrm{t}_{0}\right)\right| d u\right\}\left|\chi\left(w \mathrm{t}_{0}-\mathrm{k}\right)\right| \\
& :=\Sigma_{1}+\Sigma_{2} .
\end{aligned}
$$

About the first sum, for $\mathrm{k} \in \mathbb{Z}^{N}$ such that $\left\|\mathrm{k}-w \mathrm{t}_{0}\right\| \leq \frac{w \delta}{2}$, there holds

$$
\left\|\left(\frac{\mathrm{k}_{j}^{\prime}}{w}, u\right)-\mathrm{t}_{0}\right\| \leq\left\|\left(\frac{\mathrm{k}_{j}^{\prime}}{w}, u\right)-\frac{\mathrm{k}}{w}\right\|+\left\|\frac{\mathrm{k}}{w}-\mathrm{t}_{0}\right\|<\delta,
$$

for sufficiently large $w>0$. Therefore, by (2) and ( $\left.\chi_{2}\right), \Sigma_{1}<\frac{\epsilon}{2}$.

About $\Sigma_{2}$ notice that (see Remark 3.3 of [28]), by $\left(\chi_{2}\right), \lim _{R \rightarrow+\infty} \sum_{\|\mathrm{u}-\mathrm{k}\|>R}|\chi(\mathrm{u}-\mathrm{k})|=$ 0 uniformly on $u \in \mathbb{R}^{N}$, and hence, for sufficiently large $w>0, \sum_{\|w t-k\|>\frac{w \delta}{2}}|\chi(\mathrm{u}-\mathrm{k})|<$ $\frac{\epsilon}{4\|f\|_{\infty}}$ (without any loss of generality, $\|f\|_{\infty}>0$ ). This implies that $\Sigma_{2}<\frac{\epsilon}{2}$ and the proof is complete.

We now prove the uniform convergence result for the mixed sampling-Kantorovich operators $\left\{K_{w, j} f\right\}_{w>0}$, for every $j=1, \ldots, N$.

Theorem 2 Let $\chi$ be a kernel such that $\chi \in C\left(\mathbb{R}^{N}\right)$. If $f \in C\left(\mathbb{R}^{N}\right)$ then, for every $j=$ $1, \ldots, N, K_{w, j} f \in C\left(\mathbb{R}^{N}\right)$ and

$$
\lim _{w \rightarrow+\infty}\left\|K_{w, j} f-f\right\|_{\infty}=0 .
$$


Proof Let us fix $\epsilon>0$. By the uniform convergence of the series $\sum_{\mathrm{k} \in \mathbb{Z}^{N}}|\chi(\mathrm{u}-\mathrm{k})|$ on $[-2,2]^{N}$, for example, there exists $k_{0} \in \mathbb{Z}$ such that, for every $\mathrm{u} \in[-2,2]^{N}$,

$$
\sum_{\|\mathrm{k}\|>k_{0}}|\chi(\mathrm{u}-\mathrm{k})|<\frac{\epsilon}{3\|f\|_{\infty}} .
$$

Since $\chi \in C\left(\mathbb{R}^{N}\right)$ by assumption, there exists $\delta=\delta(\epsilon)>0$ such that, for every $t, t^{0} \in \mathbb{R}^{N}$ with $\left\|t-t^{0}\right\|<\delta$, then

$$
\left|\chi(\mathrm{t})-\chi\left(\mathrm{t}^{0}\right)\right|<\frac{\epsilon}{3\left(2 k_{0}+1\right)^{N}\|f\|_{\infty}} .
$$

Now (similarly to [21]) define $\bar{\delta}:=\min \left\{\frac{\delta}{w}, \frac{1}{w}\right\}$ and consider $t, t^{0} \in \mathbb{R}^{N}$ with $\left\|t-t^{0}\right\|<$ $\bar{\delta}$ : then $w t^{0}-\left[w t^{0}\right] \in[-2,2]^{N}$ and $w t-\left[w t^{0}\right]=w\left(t-t^{0}\right)+w t^{0}-\left[w t^{0}\right] \in[-2,2]^{N}$, and therefore, putting $\mathrm{n}:=\mathrm{k}-\left[w \mathrm{t}^{0}\right] \in \mathbb{Z}^{N}$,

$$
\begin{aligned}
& \sum_{k \in \mathbb{Z}^{N}}\left|\chi(w t-\mathrm{k})-\chi\left(w \mathrm{t}^{0}-\mathrm{k}\right)\right| \\
& \leq\left(\sum_{\|\mathrm{n}\| \leq k_{0}}+\sum_{\|\mathrm{n}\|>k_{0}}\right)\left|\chi\left(w t-\left[w \mathrm{t}^{0}\right]-\mathrm{n}\right)-\chi\left(w t_{0}-\left[w \mathrm{t}^{0}\right]-\mathrm{n}\right)\right| \\
& \leq \sum_{\|\mathrm{n}\| \leq k_{0}} \frac{\epsilon}{3\left(2 k_{0}+1\right)^{N}\|f\|_{\infty}}+2 \frac{\epsilon}{3\|f\|_{\infty}} \leq \frac{\epsilon}{\|f\|_{\infty}},
\end{aligned}
$$

by (4) and (5). Therefore we conclude that

$$
\left|\left(K_{w, j} f\right)(\mathrm{t})-\left(K_{w, j} f\right)\left(\mathrm{t}_{0}\right)\right| \leq\|f\|_{\infty} \sum_{\mathrm{k} \in \mathbb{Z}^{N}}\left|\chi(w \mathrm{t}-\mathrm{k})-\chi\left(w \mathrm{t}_{0}-\mathrm{k}\right)\right|<\epsilon
$$

for every $t, t^{0} \in \mathbb{R}^{N}$ with $\left\|t-t^{0}\right\|<\bar{\delta}$, that is, $K_{w, j} f \in C\left(\mathbb{R}^{N}\right)$, for every $w>0$, $j=1, \ldots, N$, taking into account that $\left(K_{w, j} f\right)$ is bounded (see (1)).

Now, to prove that $\lim _{w \rightarrow+\infty}\left\|K_{w, j} f-f\right\|_{\infty}=0$ it is sufficient to follow the proof of Theorem 1 taking into account of the uniform continuity of $f$.

\section{Estimates and order of approximation}

In this section we first establish a quantitative estimate for the above operators. We recall that

$$
\omega(f, \delta):=\sup \left\{|f(\mathrm{x})-f(\mathrm{y})|:\|\mathrm{x}-\mathrm{y}\| \leq \delta, \mathrm{x}, \mathrm{y} \in \mathbb{R}^{N}\right\}, \quad \delta>0,
$$

denotes the usual modulus of continuity of the function $f$. It is well-known that, if $f \in C\left(\mathbb{R}^{N}\right)$ then $\omega(f, \delta) \rightarrow 0$ as $\delta \rightarrow 0^{+}$, and moreover, the following estimate there holds

$$
\omega(f, \lambda \delta) \leq(1+\lambda) \omega(f, \delta), \quad \lambda>0 .
$$

Now we are able to prove the following result.

Theorem 3 Let $\chi$ be a kernel that satisfies the following assumption:

$$
M_{1}(\chi):=\sup _{t \in \mathbb{R}^{N}} \sum_{\mathrm{k} \in \mathbb{Z}^{N}}|\chi(\mathrm{t}-\mathrm{k})| \cdot\|t-\mathrm{k}\|<+\infty .
$$


Then, for any $f \in C\left(\mathbb{R}^{N}\right)$ there holds

$$
\left|\left(K_{w, j} f\right)(\mathrm{t})-f(\mathrm{t})\right| \leq \omega(f, 1 / w)\left\{\frac{3}{2} M_{0}(\chi)+M_{1}(\chi)\right\},
$$

$w>0,1 \leq j \leq N$

Proof Proceeding as in the proof of Theorem 1 , for every $t \in \mathbb{R}^{N}$ we can write $\left|\left(K_{w, j} f\right)(\mathrm{t})-f(\mathrm{t})\right| \leq \sum_{\mathrm{k} \in \mathbb{Z}^{N}}\left\{w \int_{\frac{k_{j}}{w}}^{\frac{k_{j}+1}{w}}\left|f\left(\frac{k_{1}}{w}, \ldots, u \ldots, \frac{k_{N}}{w}\right)-f(\mathrm{t})\right| d u\right\}|\chi(w \mathrm{t}-\mathrm{k})|$, $w>0$. By using inequality (6) it is easy to see that

$$
\begin{aligned}
& \left|\left(K_{w, j} f\right)(\mathrm{t})-f(\mathrm{t})\right| \leq \sum_{\mathrm{k} \in \mathbb{Z}^{N}}\left\{w \int_{\frac{k_{j}}{w}}^{\frac{k_{j}+1}{w}} \omega\left(f,\left\|\left(\frac{\mathrm{k}_{j}^{\prime}}{w}, u\right)-\mathrm{t}\right\|\right) d u\right\}|\chi(w \mathrm{t}-\mathrm{k})|, \\
& \leq \omega(f, 1 / w) \sum_{\mathrm{k} \in \mathbb{Z}^{N}}\left\{w \int_{\frac{k_{j}}{w}}^{\frac{k_{j}+1}{w}}\left(1+w\left\|\left(\frac{\mathrm{k}_{j}^{\prime}}{w}, u\right)-\mathrm{t}\right\|\right) d u\right\}|\chi(w \mathrm{t}-\mathrm{k})| \\
& \leq \omega(f, 1 / w) \sum_{\mathrm{k} \in \mathbb{Z}^{N}}|\chi(w \mathrm{t}-\mathrm{k})|\left\{1+w^{2} \int_{\frac{k_{j}}{w}}^{\frac{k_{j}+1}{w}}\left\|\left(\frac{\mathrm{k}_{j}^{\prime}}{w}, u\right)-\frac{\mathrm{k}}{w}\right\| d u+\|\mathrm{k}-w \mathrm{t}\|\right\} \\
& \quad=\omega(f, 1 / w) \sum_{\mathrm{k} \in \mathbb{Z}^{N}}|\chi(w \mathrm{t}-\mathrm{k})|\left\{1+w^{2} \int_{\frac{k_{j}}{w}}^{\frac{k_{j}+1}{w}}\left|u-k_{j} / w\right| d u+\|\mathrm{k}-w \mathrm{t}\|\right\} \\
& \leq \omega(f, 1 / w)\left\{\frac{3}{2} M_{0}(\chi)+M_{1}(\chi)\right\},
\end{aligned}
$$

$w>0$. This completes the proof.

Note that the requirement of Theorem 3 about the finiteness of the discrete absolute moment $M_{1}(\chi)$ is quite standard and not restrictive. Indeed in general, if we define

$$
M_{j}(\chi):=\sup _{\mathrm{t} \in \mathbb{R}^{N}} \sum_{\mathrm{k} \in \mathbb{Z}^{N}}|\chi(\mathrm{t}-\mathrm{k})|\|\mathrm{t}-\mathrm{k}\|^{j}, \quad j>0
$$

and $\chi$ is a kernel such that $\chi(t)=\mathcal{O}\left(\|t\|^{-r-1-\varepsilon}\right)$, as $\|t\| \rightarrow+\infty$, for some $r>0, \varepsilon>0$, it turns out that

$$
M_{j}(\chi)<+\infty, \quad 0 \leq j \leq r
$$

(see [30]).

Now, recalling the definition of the spaces

$$
\operatorname{Lip}(\alpha):=\left\{f \in C\left(\mathbb{R}^{N}\right): \omega(f, \delta)=\mathcal{O}\left(\delta^{\alpha}\right), \text { as } \delta \rightarrow 0^{+}\right\},
$$

for $0<\alpha \leq 1$, as a consequence of Theorem 3, we can immediately obtain the following corollary.

Corollary 1 Under the assumptions of Theorem 3 and for every $f \in \operatorname{Lip}(\alpha), 0<\alpha \leq 1$, it turns out that: 


$$
\left\|K_{w, j} f-f\right\|_{\infty}=\mathcal{O}\left(w^{-\alpha}\right), \quad \text { as } \quad w \rightarrow+\infty
$$

$j=1, \ldots, N$.

Now, we aim to prove an asymptotic expansion for the above operators. First of all we recall that, for a function $f: \mathbb{R}^{N} \rightarrow \mathbb{R}$, we denote by

$$
D^{\mathrm{h}} f:=\frac{\partial^{|\mathrm{h}|}}{\partial \mathrm{x}^{\mathrm{h}}} f=\frac{\partial^{|\mathrm{h}|}}{\partial x_{1}^{h_{1}} \ldots \partial x_{1}^{h_{N}}} f \quad\left(|\mathrm{~h}|=h_{1}+\cdots+h_{N}=r\right)
$$

the $r$-th order derivatives of $f, \mathrm{~h} \in \mathbb{N}_{0}^{N}$.

In order to reach the above aim, we need the following multivariate version of the Taylor formula for $f \in C^{r}\left(\mathbb{R}^{N}\right), r \in \mathbb{N}$ :

$$
f(\mathrm{u})=f(\mathrm{t})+\sum_{\nu=1}^{r} \sum_{|\mathrm{h}|=\nu} \frac{D^{\mathrm{h}} f(\mathrm{t})}{\mathrm{h} !}(\mathrm{u}-\mathrm{t})^{\mathrm{h}}+R_{r}(\mathrm{u} ; \mathrm{t}), \quad \mathrm{u}, \mathrm{t} \in \mathbb{R}^{N},
$$

where the term $R_{r}(\mathrm{u}, \mathrm{t})$ denotes a suitable remainder, and

$$
\mathrm{h} !:=h_{1} ! h_{2} ! \ldots h_{N} !
$$

Now, for any given kernel $\chi$, the multivariate algebraic moments, for $v \in \mathbb{N}$ and $\mathrm{h}=$ $\left(h_{1}, \ldots, h_{N}\right)$ with $|\mathrm{h}|=v$, are defined as

$$
m_{\mathrm{h}}^{v}(\chi, \mathrm{u}):=\sum_{\mathrm{k} \in \mathbb{Z}^{N}} \chi(\mathrm{u}-\mathrm{k})(\mathrm{k}-\mathrm{u})^{\mathrm{h}}, \quad \mathrm{u} \in \mathbb{R}^{N} .
$$

Now, the following asymptotic expansion can be proved.

Theorem 4 Let $\chi$ be a kernel such that, for every $\gamma>0$,

$$
\lim _{w \rightarrow+\infty} \sum_{\|w t-k\|>\gamma w}|\chi(w t-\mathrm{k})| \cdot\|w t-\mathrm{k}\|^{r}=0,
$$

uniformly with respect to $t \in \mathbb{R}^{N}$, for a certain $r \in \mathbb{N}$. Moreover, we also assume that $M_{r}(\chi)<+\infty$. Then, for any $f \in C^{r}\left(\mathbb{R}^{N}\right)$ there holds:

$\left(K_{w, j} f\right)(\mathrm{t})=f(\mathrm{t})+\sum_{\nu=1}^{r} \sum_{|\mathrm{h}|=v} \frac{D^{\mathrm{h}} f(\mathrm{t})}{\mathrm{h} !}\left[\prod_{i=1}^{N} w^{-h_{i}}\right] \sum_{\ell=0}^{h_{j}}\left(\begin{array}{c}h_{j} \\ \ell\end{array}\right) \frac{m_{\left(\mathrm{h}_{j}^{\prime}, \ell\right)}^{\nu-h_{j}+\ell}(\chi, w \mathrm{t})}{\left(h_{j}-\ell+1\right)}+o\left(w^{-r}\right)$,

as $w \rightarrow+\infty, t \in \mathbb{R}^{N}, j=1, \ldots, N$.

Proof Expanding $f\left(\frac{\mathrm{k}_{j}^{\prime}}{w}, u\right)$ by the Taylor formula (7) with remainder of the form

$$
R_{r}\left(\left(\frac{\mathrm{k}_{j}^{\prime}}{w}, u\right) ; \mathrm{t}\right)=\lambda\left(\left(\frac{\mathrm{k}_{j}^{\prime}}{w}, u\right)-\mathrm{t}\right)\left\|\left(\left(\frac{\mathrm{k}_{j}^{\prime}}{w}, u\right)-\mathrm{t}\right)\right\|^{r}
$$


where $\lambda$ is a bounded function such that $\lim _{\mathrm{v} \rightarrow 0} \lambda(\mathrm{v})=0$, we can write what follows:

$$
\begin{aligned}
& \left(K_{w, j} f\right)(\mathrm{t})=f(\mathrm{t})+\sum_{\nu=1}^{r} \sum_{|\mathrm{h}|=v} \frac{D^{\mathrm{h}} f(\mathrm{t})}{\mathrm{h} !} \sum_{\mathrm{k} \in \mathbb{Z}^{N}} \\
& \times\left[\prod_{i=1, i \neq j}^{N}\left(\frac{k_{i}}{w}-t_{i}\right)^{h_{i}} w \int_{k_{j} / w}^{\left(k_{j}+1\right) / w}\left(u-t_{j}\right)^{h_{j}} d u\right] \chi(w \mathrm{t}-\mathrm{k}) \\
& +\sum_{\mathrm{k} \in \mathbb{Z}^{N}} \chi(w \mathrm{t}-\mathrm{k})\left[w \int_{k_{j} / w}^{\left(k_{j}+1\right) / w} \lambda\left(\left(\frac{\mathrm{k}_{j}^{\prime}}{w}, u\right)-\mathrm{t}\right)\left\|\left(\left(\frac{\mathrm{k}_{j}^{\prime}}{w}, u\right)-\mathrm{t}\right)\right\|^{r} d u\right]=: f(\mathrm{t})+I_{1}+I_{2} .
\end{aligned}
$$

For $0 \leq h_{j} \leq r$ we have:

$$
\begin{aligned}
& w \int_{k_{j} / w}^{\left(k_{j}+1\right) / w}\left(u-t_{j}\right)^{h_{j}} d u=w \sum_{\ell=0}^{h_{j}}\left(\begin{array}{c}
h_{j} \\
\ell
\end{array}\right)\left(\frac{k_{j}}{w}-t_{j}\right)^{\ell} \int_{k_{j} / w}^{\left(k_{j}+1\right) / w}\left(u-\frac{k_{j}}{w}\right)^{h_{j}-\ell} d u \\
& =\sum_{\ell=0}^{h_{j}}\left(\begin{array}{c}
h_{j} \\
\ell
\end{array}\right)\left(\frac{k_{j}}{w}-t_{j}\right)^{\ell} \frac{w^{-h_{j}+\ell}}{\left(h_{j}-\ell+1\right)}=w^{-h_{j}} \sum_{\ell=0}^{h_{j}}\left(\begin{array}{c}
h_{j} \\
\ell
\end{array}\right) \frac{\left(k_{j}-w t_{j}\right)^{\ell}}{\left(h_{j}-\ell+1\right)}
\end{aligned}
$$

Hence for $I_{1}$ we get

$$
\begin{aligned}
I_{1}= & \sum_{\nu=1}^{r} \sum_{|\mathrm{h}|=v} \frac{D^{\mathrm{h}} f(\mathrm{t})}{\mathrm{h} !} w^{-h_{j}} \sum_{\ell=0}^{h_{j}}\left(\begin{array}{c}
h_{j} \\
\ell
\end{array}\right) \sum_{\mathrm{k} \in \mathbb{Z}^{N}}\left[\frac{\left(k_{j}-w t_{j}\right)^{\ell} \prod_{i=1, i \neq j}^{N}\left(\frac{k_{i}}{w}-t_{i}\right)^{h_{i}}}{\left(h_{j}-\ell+1\right)}\right] \chi(w \mathrm{t}-\mathrm{k}) \\
= & \sum_{\nu=1}^{r} \sum_{|\mathrm{h}|=v} \frac{D^{\mathrm{h}} f(\mathrm{t})}{\mathrm{h} !}\left[\prod_{i=1}^{N} w^{-h_{i}}\right] \\
& \times \sum_{\ell=0}^{h_{j}}\left(\begin{array}{c}
h_{j} \\
\ell
\end{array}\right) \frac{1}{\left(h_{j}-\ell+1\right)} \sum_{\mathrm{k} \in \mathbb{Z}^{N}}\left\{\left(k_{j}-w t_{j}\right)^{\ell} \prod_{i=1, i \neq j}^{N}\left(k_{i}-w t_{i}\right)^{h_{i}}\right\} \chi(w \mathrm{t}-\mathrm{k}),
\end{aligned}
$$

and finally

$$
I_{1}=\sum_{\nu=1}^{r} \sum_{|\mathrm{h}|=\nu} \frac{D^{\mathrm{h}} f(\mathrm{t})}{\mathrm{h} !}\left[\prod_{i=1}^{N} w^{-h_{i}}\right] \sum_{\ell=0}^{h_{j}}\left(\begin{array}{c}
h_{j} \\
\ell
\end{array}\right) \frac{m_{\left(\mathrm{h}_{j}^{\prime}, \ell\right)}^{\nu-h_{j}+\ell}(\chi, w \mathrm{t})}{\left(h_{j}-\ell+1\right)}
$$

Now we can estimate the remainder term $I_{2}$. Since $\lim _{\mathrm{v} \rightarrow 0} \lambda(\mathrm{v})=0$ for a fixed $\varepsilon>0$ there exists $\gamma>0$ such that, for $\|\mathrm{v}\| \leq \gamma$ there holds $|\lambda(\mathrm{v})|<\varepsilon$. Thus:

$$
\begin{aligned}
I_{2}= & \left\{\sum_{\|w t-\mathrm{k}\| \leq w \gamma / 2}+\sum_{\|w \mathrm{t}-\mathrm{k}\|>w \gamma / 2}\right\} \chi(w \mathrm{t}-\mathrm{k}) \\
& \times\left[w \int_{k_{j} / w}^{\left(k_{j}+1\right) / w} \lambda\left(\left(\frac{\mathrm{k}_{j}^{\prime}}{w}, u\right)-\mathrm{t}\right)\left\|\left(\left(\frac{\mathrm{k}_{j}^{\prime}}{w}, u\right)-\mathrm{t}\right)\right\|^{r} d u\right] \\
= & : I_{2,1}+I_{2,2} .
\end{aligned}
$$

Concerning $I_{2,1}$, for every $\mathrm{k} \in \mathbb{Z}^{N}$ such that $\|w t-\mathrm{k}\| \leq w \gamma / 2$ and $u \in\left[k_{j} / w,\left(k_{j}+1\right) / w\right]$ :

$$
\left\|\left(\left(\frac{\mathrm{k}_{j}^{\prime}}{w}, u\right)-\mathrm{t}\right)\right\| \leq\left\|\left(\left(\frac{\mathrm{k}_{j}^{\prime}}{w}, u\right)-\frac{\mathrm{k}}{w}\right)\right\|+\|\mathrm{k} / w-\mathrm{t}\| \leq\left|u-\frac{k_{j}}{w}\right|+\frac{\gamma}{2}<\gamma,
$$


for $w>0$ sufficiently large, hence:

$$
\begin{aligned}
\left|I_{2,1}\right| & <\varepsilon \sum_{\|w \mathrm{t}-\mathrm{k}\| \leq w \gamma / 2}|\chi(w \mathrm{t}-\mathrm{k})|\left[w \int_{k_{j} / w}^{\left(k_{j}+1\right) / w}\left\|\left(\left(\frac{\mathrm{k}_{j}^{\prime}}{w}, u\right)-\mathrm{t}\right)\right\|^{r} d u\right] \\
& \leq \varepsilon 2^{r-1} \sum_{\|w \mathrm{t}-\mathrm{k}\| \leq w \gamma / 2}|\chi(w \mathrm{t}-\mathrm{k})|\left[w \int_{k_{j} / w}^{\left(k_{j}+1\right) / w}\left\|\left(\frac{\mathrm{k}_{j}^{\prime}}{w}, u\right)-\frac{\mathrm{k}}{\mathrm{w}}\right\|^{r} d u+\left\|\frac{\mathrm{k}}{\mathrm{w}}-\mathrm{t}\right\|^{r}\right] \\
& \leq \varepsilon 2^{r-1} \sum_{\|w \mathrm{t}-\mathrm{k}\| \leq w \gamma / 2}|\chi(w \mathrm{t}-\mathrm{k})|\left[\frac{w^{-r}}{r+1}+w^{-r}\|w \mathrm{t}-\mathrm{k}\|^{r}\right] \\
& \leq \varepsilon w^{-r} 2^{r-1}\left[\frac{M_{0}(\chi)}{r+1}+M_{r}(\chi)\right]<+\infty,
\end{aligned}
$$

for $w>0$ sufficiently large. Furthermore, by exploiting the above computations, and by using assumption (8) we have:

$$
\begin{aligned}
\left|I_{2,2}\right| & \leq\|\lambda\|_{\infty} \sum_{\|w t-\mathrm{k}\|>w \gamma / 2}|\chi(w \mathrm{t}-\mathrm{k})|\left[w \int_{k_{j} / w}^{\left(k_{j}+1\right) / w}\left\|\left(\left(\frac{\mathrm{k}_{j}^{\prime}}{w}, u\right)-\mathrm{t}\right)\right\|^{r} d u\right] \\
& \leq\|\lambda\|_{\infty} 2^{r-1} w^{-r} \sum_{\|w \mathrm{t}-\mathrm{k}\|>w \gamma / 2}|\chi(w \mathrm{t}-\mathrm{k})|\left[\frac{1}{r+1}+\|w \mathrm{t}-\mathrm{k}\|^{r}\right] \\
& \leq\|\lambda\|_{\infty} 2^{r-1} w^{-r}\left[\left(\frac{2}{\gamma w}\right)^{r} \frac{1}{r+1}+1\right] \sum_{\|w t-\mathrm{k}\|>w \gamma / 2}|\chi(w \mathrm{t}-\mathrm{k})| \cdot\|w \mathrm{t}-\mathrm{k}\|^{r} \\
& <\|\lambda\|_{\infty} 2^{r} w^{-r} \varepsilon,
\end{aligned}
$$

for $w>0$ sufficiently large. This completes the proof.

Now, from Theorem 4 it is easy to establish the following Voronovskaja type theorem.

Theorem 5 Under the assumption of Theorem 4 with $r=1$, if we assume in addition that the following algebraic moments:

$$
m_{e_{i}}^{1}(\chi, \mathrm{u})=A_{e_{i}}^{1} \in \mathbb{R}, \quad \mathrm{u} \in \mathbb{R}^{N},
$$

are constants, where $e_{i}:=(0, \ldots, 0,1,0, \ldots 0), i=1, \ldots, N$, we have:

$$
\lim _{w \rightarrow+\infty} w\left\{\left(K_{w, j} f\right)(\mathrm{t})-f(\mathrm{t})\right\}=\sum_{i=1, i \neq j}^{N} \frac{\partial^{1}}{\partial x_{i}} f(\mathrm{t}) \cdot A_{e_{i}}^{1}+\frac{\partial^{1}}{\partial x_{j}} f(\mathrm{t})\left[\frac{1}{2}+A_{e_{j}}^{1}\right],
$$

$\mathrm{t} \in \mathbb{R}^{N}, 1 \leq j \leq N$

Proof By Theorem 4 in the case of $r=1$ we know:

$$
w\left\{\left(K_{w, j} f\right)(\mathrm{t})-f(\mathrm{t})\right\}=\sum_{i=1, i \neq j}^{N} \frac{\partial^{1}}{\partial x_{i}} f(\mathrm{t}) \cdot A_{e_{i}}^{1}+\frac{\partial^{1}}{\partial x_{j}} f(\mathrm{t})\left[\frac{1}{2}+A_{e_{j}}^{1}\right]+w o\left(w^{-1}\right),
$$

then the proof follows immediately by passing to the limit for $w \rightarrow+\infty$.

It can be useful to observe that the moment-type assumptions required in Theorems 4 and Theorem 5 are quite standard and are satisfied by several examples of kernels, such as those mentioned in Sect. 2. A wide list of them can be found, e.g., in [2,14,16,22]. 


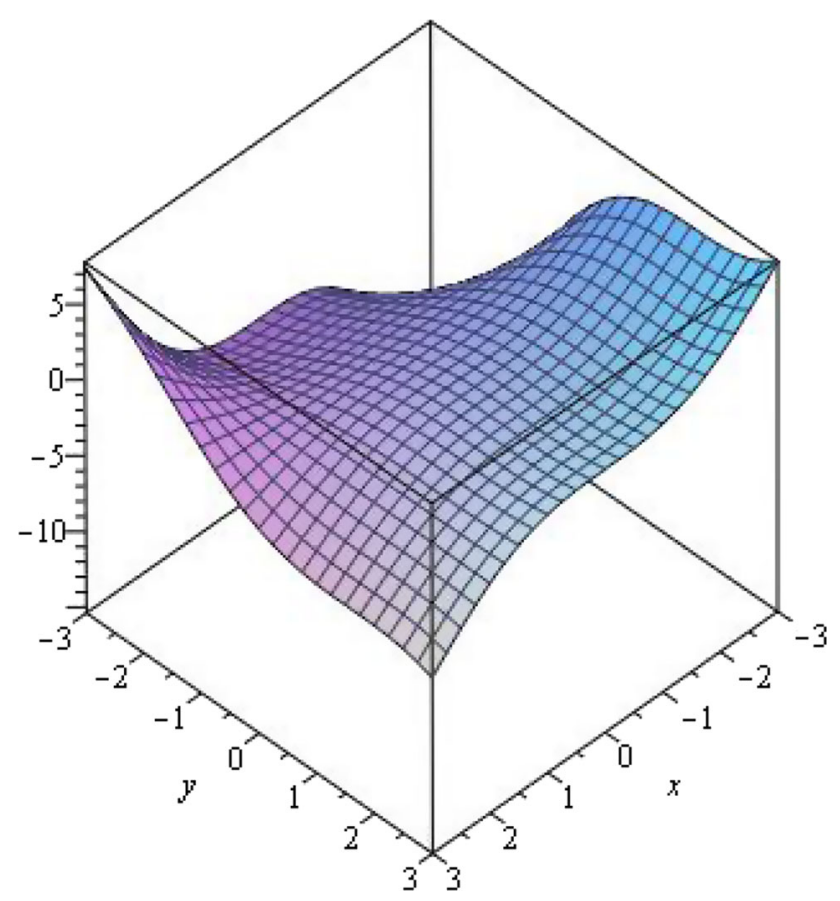

Fig. 1 The plot of the bi-dimensional function $f$
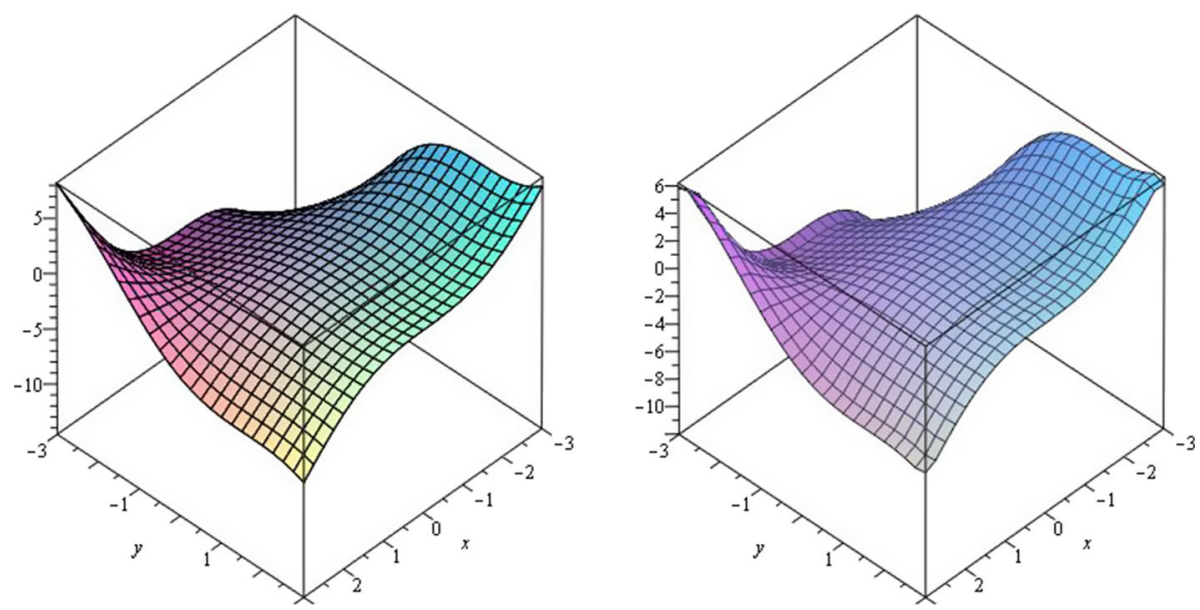

Fig. 2 On the left, we have the operator $K_{w, 1} f$, with $w=5$, while on the right we have the operator $K_{w, 1} f$, with $w=10$, both based upon the bi-dimensional central B-spline of order 3

Remark 1 Note that a quantitative version of Theorems 4 and 5 can be easily established by repeating the above proof using the Lagrange remainder in the Taylor expansion (7) and by using some well-known inequalities; for more details see, e.g., $[1,16]$.

Further, we can also observe that Theorem 5 can be also generalized for higher orders (i.e., $r>1$ ). In order to get such generalization, we must require that the algebraic moments 

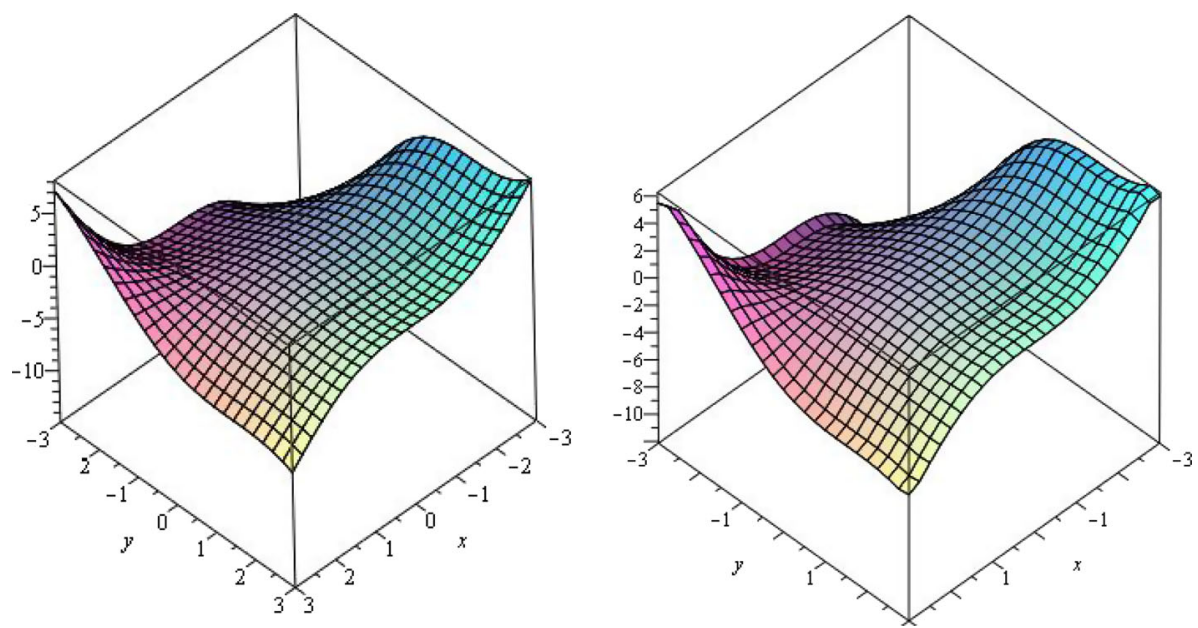

Fig. 3 On the left, we have the operator $K_{w, 2} f$, with $w=5$, while on the right we have the operator $K_{w, 2} f$, with $w=10$, both based upon the bi-dimensional central B-spline of order 3

of the kernel $\chi$ :

$$
m_{\mathrm{h}}^{\nu}(\chi, \mathrm{u})=A_{\mathrm{h}}^{\nu} \in \mathbb{R}, \quad \mathrm{u} \in \mathbb{R}^{N},
$$

are constants, for every $v=1, \ldots, r$, and $\mathrm{h} \in \mathbb{N}^{N}$ with $|\mathrm{h}|=v$, and that

$$
\sum_{\ell=0}^{h_{j}}\left(\begin{array}{c}
h_{j} \\
\ell
\end{array}\right) \frac{A_{\left(\mathrm{h}_{j}^{\prime}, \ell\right)}^{\nu-h_{j}+\ell}}{\left(h_{j}-\ell+1\right)}=0,
$$

for all $v=1, \ldots, r-1$, and $\mathrm{h}=\left(h_{1}, \ldots, h_{N}\right) \in \mathbb{N}^{N}$ with $|\mathrm{h}|=v, j=1, \ldots, N$. Examples of kernels satisfying the above conditions can be generated using product kernels, in which the one-dimensional factors are given by suitable finite linear combination of wellknown univariate kernels. The procedure for the construction of such multivariate functions is analogous to that one used in [16].

Finally, we give a numerical example showing the approximations that can be achieved by the above theory. Consider the function $f\left(x_{1}, x_{2}\right):=\left(\sin x_{1}-x_{1}+1\right)\left(x_{2}+\cos x_{2}\right)$, $\left(x_{1}, x_{2}\right) \in \mathbb{R}^{2}$ (see Fig. 1). The approximations obtained by the mixed sampling-Kantorovich operators based upon the bi-dimensional central B-spline of order 3 are depicted in Figs. 2 and 3 .

Acknowledgements The authors are members of the Gruppo Nazionale per l'Analisi Matematica, la Probabilitá e le loro Applicazioni (GNAMPA) of the Istituto Nazionale di Alta Matematica (INdAM), of RITA (Research ITalian network on Approximation) and of the UMI group "Teoria dell'Approssimazione e Applicazioni". The authors are partially supported by the "Department of Mathematics and Computer Science" of the University of Perugia (Italy) and within the projects "Metodi e processi innovativi per lo sviluppo di una banca di immagini mediche per fini diagnostici" (2018) and "Metodiche di Imaging non invasivo mediante angiografia OCT sequenziale per lo studio delle Retinopatie degenerative dell' Anziano (M.I.R.A.)" (2019) funded by the Fondazione Cassa di Risparmio di Perugia. The first and the third author have been partially supported within the projects "Metodi di Teoria dell'Approssimazione, Analisi Reale, Analisi Nonlineare e loro applicazioni" and "Integrazione, Approssimazione, Analisi Nonlineare e loro Applicazioni", funded by the 2018 and 2019 basic research fund of the University of Perugia. Finally, the first and the second author of the paper have been partially supported within a 2019 GNAMPA-INdAM Project ("Metodi di analisi reale 
per l'approssimazione attraverso operatori discreti e applicazioni”) and a 2020 GNAMPA-INdAM Project ("Analisi reale, teoria della misura ed approssimazione per la ricostruzione di immagini").

Funding Open access funding provided by Università degli Studi di Perugia within the CRUI-CARE Agreement.

Open Access This article is licensed under a Creative Commons Attribution 4.0 International License, which permits use, sharing, adaptation, distribution and reproduction in any medium or format, as long as you give appropriate credit to the original author(s) and the source, provide a link to the Creative Commons licence, and indicate if changes were made. The images or other third party material in this article are included in the article's Creative Commons licence, unless indicated otherwise in a credit line to the material. If material is not included in the article's Creative Commons licence and your intended use is not permitted by statutory regulation or exceeds the permitted use, you will need to obtain permission directly from the copyright holder. To view a copy of this licence, visit http://creativecommons.org/licenses/by/4.0/.

\section{References}

1. Acar, T., Aral, A., Rasa, I.: Approximation by k-th order modifications of Szaás-Mirakyan operators. Stud. Sci. Math. Hungar. 53(3), 379-398 (2016)

2. Acar, T., Costarelli, D., Vinti, G.: Linear prediction and simultaneous approximation by $\mathrm{m}$-th order Kantorovich type sampling series. Banach J. Math. Anal. 14(4), 1481-1508 (2020)

3. Agrawal, P.N., Acu, A.M., Sidharth, M.: Approximation degree of a Kantorovich variant of Stancu operators based on Pólya-Eggenberger distribution. Rev. R. Acad. Cienc. Exactas Fis. Nat. Ser. A Mat. RACSAM 113(1), 137-156 (2019)

4. Anastassiou, G.A.: Quantitative approximation by perturbed Kantorovich-Choquet neural network operators. Rev. R. Acad. Cienc. Exactas Fis. Nat. Ser. A Mat. RACSAM 113, 875-900 (2019)

5. Angeloni, L., Costarelli, D., Vinti, G.: A characterization of the convergence in variation for the generalized sampling series. Ann. Acad. Sci. Fenn. Math. 43, 755-767 (2018)

6. Angeloni, L., Costarelli, D., Vinti, G.: A characterization of the absolute continuity in terms of convergence in variation for the sampling Kantorovich operators. Mediterr. J. Math. 16, 44 (2019). https://doi.org/10. 1007/s00009-019-1315-0

7. Angeloni, L., Costarelli, D., Vinti, G.: Convergence in variation for the multidimensional generalized sampling series and applications to smoothing for digital image processing. Ann. Acad. Sci. Fenn. Math. 45, 751-770 (2020)

8. Angeloni, L., Costarelli, D., Vinti, G.: Quantitative estimates for sampling type operators with respect to the Jordan variation. Atti Accad. Naz. Lincei Rendiconti Lincei Mat. Appl. 31, 269-284 (2020)

9. Angeloni, L., Vinti, G.: Approximation with respect to Goffman-Serrin variation by means of nonconvolution type integral operators. Numer. Funct. Anal. Optim. 31, 519-548 (2010)

10. Angeloni, L., Vinti, G.: A sufficient condition for the convergence of a certain modulus of smoothness in multidimensional setting. Comm. Appl. Nonlinear Anal. 20(1), 1-20 (2013)

11. Angeloni, L., Vinti, G.: A characterization of absolute continuity by means of Mellin integral operators. Z. Anal. Anwend 34(3), 343-356 (2015)

12. Angeloni, L., Vinti, G.: Convergence in variation and a characterization of the absolute continuity. Integral Transforms Spec. Funct. 26(10), 829-844 (2015)

13. Angeloni, L., Vinti, G.: Discrete operators of sampling-type and approximation in $\phi$-variation. Math. Nachr. 291, 546-555 (2018)

14. Asdrubali, F., Baldinelli, G., Bianchi, F., Costarelli, D., Rotili, A., Seracini, M., Vinti, G.: Detection of thermal bridges from thermographic images by means of image processing approximation algorithms. Appl. Math. Comput. 317, 160-171 (2018)

15. Bardaro, C., Butzer, P.L., Stens, R.L., Vinti, G.: Kantorovich-type generalized sampling series in the setting of Orlicz spaces. Sampling Theory Signal Image Process. 6, 29-52 (2007)

16. Bardaro, C., Mantellini, I.: On pointwise approximation properties of multivariate semi-discrete sampling type operators. Results Math. 72, 1449-1472 (2017)

17. Bardaro, C., Mantellini, I., Stens, R., Vautz, J., Vinti, G.: Generalized Sampling Approximation for Multivariate Discontinuous Signals and Application to Image Processing. New Perspectives on Approximation and Sampling Theory-Festschrift in Honor of Paul Butzer's 85th Birthday, pp. 87-114. Birkhauser, Basel (2014) 
18. Boche, H., Mönich, U.J., Tampubolon, E.: Spaceability and strong divergence of the Shannon sampling series and applications. J. Approx. Theory 222, 157-174 (2017)

19. Buhmann, M.D., De Marchi, S., Perracchione, E.: Analysis of a new class of rational RBF expansions. IMA J. Numer. Anal. 40, 1972-1993 (2020)

20. Butzer, P.L., Fisher, A., Stens, R.L.: Generalized sampling approximation of multivariate signals: theory and applications. Note Mat. 10(1), 173-191 (1990)

21. Butzer, P.L., Fisher, A., Stens, R.L.: Generalized sampling approximation of multivariate signals: general theory. Atti Sem. Mat. Fis. Univ. Modena 41(1), 17-37 (1993)

22. Butzer, P.L., Nessel, R.J.: Fourier Analysis and Approximation I. Academic Press, New York (1971)

23. Butzer, P.L., Splettstößer, W.: A sampling theorem for duration limited functions with error estimates. Inform. Contr. 34, 55-65 (1977)

24. Butzer, P.L., Splettstößer, W., Stens, R.L.: The sampling theorem and linear prediction in signal analysis. Jahresber. Deutsch. Math.-Verein. 90, 1-70 (1988)

25. Butzer, P.L., Stens, R.L.: Linear prediction by samples from the past. Adv. Top. Shannon Sampl. Interpolat. Theory 20, 157-183 (1993)

26. Cantarini, M., Costarelli, D., Vinti, G.: A solution of the problem of inverse approximation for the sampling Kantorovich operators in case of Lipschitz functions. Dolomites Res. Notes Approx. 13, 30-35 (2020)

27. Chen, L., Zhang, H.: Sharp exponential bounds for the Gaussian regularized Whittaker-KotelnikovShannon sampling series. J. Approx. Theory 245, 73-82 (2019)

28. Costarelli, D., Vinti, G.: Approximation by Multivariate Generalized Sampling Kantorovich Operators in the Setting of Orlicz Spaces. Boll. Unione Mat. Ital. 4(3), 445-468 (2011)

29. Costarelli, D., Vinti, G.: Rate of approximation for multivariate sampling Kantorovich operators on some functions spaces. J. Integral Equ. Appl. 26(4), 455-481 (2014)

30. Costarelli, D., Vinti, G.: Inverse results of approximation and the saturation order for the sampling Kantorovich series. J. Approx. Theory 242, 64-82 (2019)

31. Costarelli, D., Vinti, G.: Saturation by the Fourier transform method for the sampling Kantorovich series based on bandlimited kernels. Anal. Math. Phys. 9, 2263-2280 (2019)

32. Costarelli, D., Vinti, G.: An inverse result of approximation by sampling Kantorovich series. Proc. Edinburgh Math. Soc. 62(1), 265-280 (2019)

33. Costarelli, D., Vinti, G.: Convergence of sampling Kantorovich operators in modular spaces with applications. Rend. Circ. Mat. Palermo (2020). https://doi.org/10.1007/s12215-020-00544-Z

34. De Marchi, S., Erb, W., Marchetti, F., Perracchione, E., Rossini, M.: Shape-driven interpolation with discontinuous kernels: error analysis, edge extraction, and applications in magnetic particle imaging. SIAM J. Sci. Comput. 42(2), B472-B491 (2020)

35. Deo, N., Dhamija, M.: D Mičauş: Stancu-Kantorovich operators based on inverse Pólya-Eggenberger distribution. Appl. Math. Comput. 273, 281-289 (2016)

36. Dyn, N., Jackson, I.R.H., Levin, D., Ron, A.: On multivariate approximation by integer translates of a basis function. Israel J. Math. 78(1), 95-130 (1992)

37. Dyn, N., Leviatan, D., Levin, D., Pinkus, A.: Multivariate Approximation and Applications. Cambridge University Press, Cambridge (2001)

38. Gal, S., Opris, B.D.: Uniform and pointwise convergence of Bernstein-Durrmeyer operators with respect to monotone and submodular set functions. J. Math. Anal. Appl. 424(2), 1374-1379 (2015)

39. Kolomoitsev, Y.S., Skopina, M.A.: Approximation by multivariate Kantorovich-Kotelnikov operators. J. Math. Anal. Appl. 456(1), 195-213 (2017)

40. Kumar, A.S., Shivam, B.: Inverse approximation and GBS of bivariate Kantorovich type sampling series. Rev. R. Acad. Cienc. Exactas Fis. Nat. Ser. A Mat. RACSAM 114(82), 15 (2020)

41. Lin, R.: An optimal convergence rate for the Gaussian regularized Shannon sampling series. Numer. Funct. Anal. Optim. 40(3), 241-258 (2019)

42. Milovanović, G.V., Mursaleen, M., Nasiruzzaman, M.: Modified Stancu type Dunkl generalization of Szász-Kantorovich operators. Rev. R. Acad. Cienc. Exactas Fis. Nat. Ser. A Mat. RACSAM 112, 135151 (2018)

43. Mohiuddine, S.A., Özger, F.: Approximation of functions by Stancu variant of Bernstein-Kantorovich operators based on shape parameter. Rev. R. Acad. Cienc. Exactas Fis. Nat. Ser. A Mat. RACSAM 114(70), 17 (2020)

44. Orlova, O., Tamberg, G.: On approximation properties of generalized Kantorovich-type sampling operators. J. Approx. Theory 201, 73-86 (2016)

45. Rahman, S., Mursaleen, M., Khan, A.: A Kantorovich variant of Lupaş-Stancu operators based on Pólya distribution with error estimation. Rev. R. Acad. Cienc. Exactas Fis. Nat. Ser. A Mat. RACSAM 114(75), 26 (2020) 
46. Smale, S., Zhou, D.X.: Shannon sampling and function reconstruction from point values. Bull. Am. Math. Soc. 41(3), 279-305 (2004)

47. Smale, S., Zhou, D.X.: Shannon sampling II: connections to learning theory. Appl. Comput. Harmonic Anal. 19(3), 285-302 (2005)

48. Wu, Q., Ying, Y., Zhou, D.X.: Multi-kernel regularized classifiers. J. Complex. 23(1), 108-134 (2007)

Publisher's Note Springer Nature remains neutral with regard to jurisdictional claims in published maps and institutional affiliations. 